\title{
Habilidades sociales en adolescentes institucionalizados: Una intervención con juego grupal
}

\section{Social skills in institutionalized teenagers: A group play intervention}

Rosa Cindy Estrada Rodríguez

Elia María Escoffié Aguilar

Ricardo García

Universidad Autónoma de Yucatán

\section{Resumen}

Las habilidades sociales están involucradas en muchas áreas de la vida de una persona, favorecen aspectos como la adaptación al medio en el que se vive y la protección de salud, tanto física como mental. El incremento de estas habilidades en adolescentes institucionalizados puede resultar especialmente beneficioso, debido a las situaciones de riesgo de las cuales suelen venir y que pueden ser causantes de otros problemas en los jóvenes. El presente trabajo es un estudio de caso, que tuvo como objetivo fomentar el uso de habilidades sociales en esta población por medio de un taller grupal de carácter lúdico. Los resultados muestran que este tipo de intervención pudiera estar relacionado con el aumento de habilidades sociales, ya que se observó un incremento en las habilidades de siete de los ocho integrantes.

Palabras clave: adolescentes institucionalizados, habilidades sociales, adaptación, juego grupal, técnicas Gestalt.

\begin{abstract}
Social skills are involved in many areas of a person's life, improving aspects such as the adaptation to their living environment and their health protection, both physical and mental. The increase of social skills in institutionalized teenagers may be particularly beneficial, because of the risk situations of the environment they come from, and that can be causes of other problems in young people. This paper, a case study, seeks to promote the use of social skills in this population through a ludic group workshop. The results show that this kind of intervention result in the increase of social skills, since an increase was observed in the skills of seven of the eight members.

Keywords: institutionalized teenagers, social skills, adjustment, group play, Gestalt techniques.

Nota del autor

Rosa Cindy Estrada Rodríguez, Facultad de Psicología, Universidad Autónoma de Yucatán (UADY); Elia María Escoffié Aguilar, Facultad de Psicología, UADY; Ricardo García, Facultad de Psicología, UADY.

La correspondencia en relación a este artículo debe dirigirse a R. Cindy Estrada Rodríguez, Facultad de Psicología, UADY, carretera Mérida-Tizimin, Km 1, Cholul. Mérida, Yucatán, México.

Dirección electrónica: psic.estrada.r@gmail.com
\end{abstract}


La adolescencia es una etapa donde el ser humano atraviesa por múltiples cambios, desde físicos hasta psicológicos, cuyo fin es preparar a la persona para la adultez. Pasar por cambios diversos puede dificultarse si no se cuenta con las herramientas personales adecuadas y con oportunidades del medio ambiente para sobrellevarlos, tales como tolerancia a la frustración, expresión adecuada de las emociones, sentimientos y pensamientos, contar con una persona de confianza, practicar un pasatiempo, entre muchas otras.

Los adolescentes institucionalizados pueden sufrir carencias en estas herramientas y oportunidades. A pesar de que la institucionalización se creó con el objetivo de brindarles un espacio seguro a los menores que se quedan sin hogar y de cubrir sus necesidades fisiológicas, entre algunas otras, las vivencias de un adolescente institucionalizado son muy diferentes a las de un adolescente que vive dentro de un núcleo familiar, por lo tanto, la socialización es una de las áreas más afectadas.

El objetivo de la investigación aplicada fue probar un programa de intervención, que fomente las habilidades sociales en adolescentes institucionalizados para facilitar la adaptación a su medio ambiente. Para esto se buscó sensibilizar a los jóvenes sobre temas que involucren las habilidades sociales y su beneficio, así como practicar estas habilidades mediante juegos y técnicas vivenciales.

La técnica utilizada para la intervención fue un taller vivencial con temas relacionados con la adquisición y la práctica de las habilidades sociales. Para ponerlas en práctica, se eligió el juego con base en las técnicas del enfoque Gestalt, que tiene características que van acordes con los objetivos del programa. Antes y después de la intervención, se aplicó una escala que sirviera de referencia para analizar, posteriormente, qué tanto la intervención había sido útil.

Cabe señalar que al ser un estudio de caso en un contexto específico, se busca utilizar y, más tarde, describir esta intervención con este tipo de población, sin buscar generalizar los resultados. Para la generalización de resultados se tendría que probar esta misma intervención en poblaciones con características diferentes.

\section{Las habilidades Sociales (HS)}

Las HS son aquellos comportamientos que nos ayudan a resolver una situación de carácter social de una manera efectiva; esto implica que la solución sea aceptable para la persona y para el ambiente donde se encuentra (Trianes, Muñoz, \& Jiménez, 1997). Sin HS sería imposible para el ser humano socializar. Caballo (2010) menciona que las HS en la infancia son de gran importancia en el proceso de socialización de cualquier persona, e incluso repercutir en el desarrollo cognoscitivo y emocional.

Según Trianes, Muñoz y Jiménez (1997), las HS tienen dos componentes, el objetivo y la estrategia, que interactúan para dar como resultado una conducta. El objetivo es la meta que la persona quiere lograr en la interacción 
con otros y, en consecuencia, guía su conducta a partir de este componente. La forma cómo va a lograr ese propósito es la estrategia, que puede ser la única forma conocida por el individuo para actuar en esa situación, aquella que ha elegido entre un repertorio de estrategias por ser la más adecuada según su parecer para determinado contexto social.

Además, las HS forman parte de la inteligencia interpersonal, que tiene impacto en el éxito de todos los aspectos de la vida de una persona, así como en la manera de afrontar situaciones difíciles, la cotidianeidad, en la toma de decisiones, entre otros aspectos (Ostrovsky, 2007).

Varios autores afirman que las HS cumplen una función adaptativa, por lo tanto, resulta de gran relevancia el entrenamiento de éstas desde edades tempranas y la intervención en adolescentes, de tal forma es posible prevenir futuras problemáticas conductuales $\mathrm{y} / \mathrm{o}$ de salud mental (Lacunza, 2009; Lacunza \& Cortini, 2011; Kelly, 2002). Otro componente importante que cumplen las habilidades sociales es su influencia en el desarrollo de fortalezas psíquicas en los niños y en la autoestima. Además de estar relacionadas con la salud física y mental (Lacunza, 2009).

\section{El adolescente institucionalizado}

Un menor de edad que vive en institución es aquél o aquélla que, a falta de cuidados parentales, es internado(a) para su resguardo dentro de un espacio que está regulado por adultos cuidadores.

Se ha encontrado en varios estudios que los menores institucionalizados presentan una serie problemas psicosociales y de alto riesgo, además de trastornos de apego, de ahí que sean una población vulnerable (Fernández \& Fernández, 2012).

Ison y Morelato $(2002 ; 2007)$ afirman que la familia es un contexto clave en el desarrollo de HS ya que sirve como referente para evaluar la conducta social, haciéndole ver al niño qué es socialmente correcto y qué no. De esta forma, en lo relacionado con el desarrollo de HS, los adolescentes institucionalizados pudieran encontrarse en desventaja en comparación con los adolescentes con cuidados parentales, que crecieron y están dentro de un ambiente familiar.

Muchas experiencias cotidianas están limitadasparalosadolescentesinstitucionalizados o, incluso, éstos no tienen acceso a ellas. Por ejemplo, la vivencia de la sexualidad, generalmente muy estricta dentro de las instituciones para la protección del adolescente, puede crear una percepción distorsionada de las relaciones entre hombres y mujeres, y problemas mayores en la comunicación con el sexo opuesto cuando se llega a la edad adulta. Otro aspecto que puede verse afectado en los adolescentes institucionalizados es el desarrollo moral, debido a los patrones de reforzamiento y castigo que se ven obligadas a utilizar las instituciones para mantener el control; dicha 
situación dificulta el razonamiento moral posterior, el pensamiento crítico y la toma de decisiones (Carcelén \& Martínez, 2008).

\section{La intervención con juego grupal}

El juego tiene muchos beneficios para la persona, sin importar la edad, ya que es divertido, creativo, alivia el estrés y brinda la oportunidad de establecer vínculos con las demás personas para experimentar y aprender; fomenta las interacciones y la comunicación social positiva. Además, tiene un impacto benéfico en el aprendizaje de la regulación de emociones y la tolerancia de la frustración (Romera, Ortega, \& Monks, 2008; Schaefer, 2012). El juego resulta una actividad importante para la adquisición de ciertos aspectos como la vinculación afectiva, habilidades comunicativas, toma de decisiones, el pensamiento creativo, etc. El juego se convierte en el mundo a su escala, para poner en práctica las competencias necesarias para la vida en sociedad (Romera et al., 2008).

Entre otras cualidades, el juego posee poderes terapéuticos que lo hacen una actividad incomparablemente beneficiosa. Estos poderes abarcan desde la comunicación, regulación emocional, mejora de la relación, juicio moral, manejo del estrés, fortalecimiento del yo, preparación para la vida $\mathrm{y}$ autorrealización (Schaefer, 2012).

Asimismo, el juego en grupo se hace aún más provechoso. Es aquí donde el papel de los pares adquiere mucha importancia. Como menciona Schaffer (2000), la función de los pares es brindar la oportunidad de aprender habilidades que sólo se pueden aprender entre ellos, como son la cooperación, la competencia, tomar turnos, compartir, etc., partiendo del supuesto de que entre ellos tienen las mismas oportunidades por ser del mismo rango de edad.

Schaffer (2010), en dicho sentido, menciona incluso la posibilidad de que entre pares se pudieran desarrollar vínculos afectivos y roles como si se tratase de relaciones con adultos, de modo que se cumplieron así papeles familiares inexistentes. Esto no se ha comprobado en niños institucionalizados, pero sí en casos en los que los niños han quedado huérfanos por situación de guerra.

El trabajo en grupo tiene la ventaja de ser un mundo a escala, privado, donde se puede vivenciar la conducta actual y ensayar las nuevas conductas que se desean aprender. Se ha dicho que el trabajo en grupo es un escenario ideal para niños, y no tan niños, que requieren practicar HS, ya que proporcionarles un espacio donde puedan interactuar con otros chicos de su edad puede ayudarlos a descubrir, mediante la presencia del otro, las habilidades personales $\mathrm{y}$ experimentar nuevas formas de relacionarse (Oaklander, 2001).

\section{El enfoque Gestalt}

El enfoque Gestalt está interesado en el funcionamiento saludable e integrado de todo el organismo, desde los sentidos, el cuerpo, las emociones y el intelecto; a través de sus técnicas busca facilitar para cada individuo su 
propio desarrollo y el encuentro de metas que le sean significativas en su proceso de maduración (Burga, 1981; Oaklander, 2008).

Dicho enfoque está relacionado con los procesos de adquisición y la práctica de las HS, ya que como menciona Burga (1981), una gran ventaja del enfoque Gestalt es su misma naturaleza, su enfoque vivencial, que fomenta la acción para el trabajo grupal. Además, instruye a la persona para prestar atención a lo que siente y desea, a hacer un contacto con su realidad, tanto externa como interna, y a vivir en el aquí y en el ahora. Asimismo, la persona va tomando responsabilidad de todos estos aspectos y de lograr cambios por sí misma, sin que alguien le diga que necesita cambiar.

De este modo, las técnicas que utiliza el enfoque Gestalt pueden mejorar el percatarse, la responsabilidad y las capacidades de escuchar, entre otras. Es de gran ayuda, tanto en el trabajo grupal como en el fomento de las HS (Oaklander, 2001). Así, gran parte de los juegos utilizados en la intervención tuvo una base en las técnicas Gestalt para adquirir los beneficios antes mencionados.

\section{Método}

\section{Participantes}

El grupo de estudio se conformó por ocho adolescentes varones de entre 13 y 17, años pertenecientes a un albergue cristiano para jóvenes varones, en la ciudad de Mérida, Yucatán.
La invitación al taller fue abierta para todos los jóvenes del albergue, se contó con la asistencia de todos los participantes en la mayoría de las sesiones. El tiempo de institucionalización variaba en cada joven, algunos tenían más de 10 años ahí, mientras otros sólo unos meses. Ninguno acudía a un sistema de educación escolarizado, recibían clases de primaria y secundaria abierta, por medio del Instituto Nacional para la Educación de los Adultos (INEA). Sus actividades cotidianas consistían en clases dentro del albergue, tareas domésticas y momentos de recreación.

\section{Instrumentos}

La herramienta utilizada para evaluar los efectos de la intervención fue The Matson Evaluation of Social Skills in Youngsters (MESSY) de Matson, Rotatori y Helsel (1983). Es un instrumento que busca evaluar el grado de adecuación de la conducta social y se compone de dos formatos: un autoinforme para el adolescente, con 62 reactivos en total, y un cuestionario para el maestro, con 64 reactivos. Para las dos versiones, las opciones de respuesta y la puntuación se clasifican en Nunca (1), Algunas veces (2), Casi siempre (3) y Siempre (4). En este estudio se utilizó la versión en español, traducida por Trianes, Blanca, Muñoz, García, Cardelle-Elawar e Infante (2002).

Para la calificación de dicha escala, Ipiña, Molina y Reyna (2011) y Trianes et al. (2002), hicieron una clasificación de los ítems en la 
versión autoinforme a partir de cinco factores, clasificados en dos grandes dimensiones:

- Habilidades Sociales Apropiadas: esta dimensión evalúa diversas conductas, como la expresión emocional, ser asertivo, tener amigos, etcétera.

- Un conjunto de cuatro factores: Asertividad Inapropiada, Impulsividad, Sobreconfianza y Celos/Soledad. Esta dimensión evalúa diversas conductas, como ser impulsivo, conductas agresivas, conductas de sobrevaloración del Yo y conductas de soledad.

En esta clasificación de Ipiña, et al. (2011), también se estudiaron los índices de consistencia interna, los cuales fueron aceptables en la mayoría de las dimensiones: factor Habilidades Sociales Apropiadas y factor Asertividad Inapropiada mostraron índices adecuados ( $\alpha=.81, .85$ y .79, respectivamente), factor Impulsividad y factor Sobreconfianza tuvieron índices aceptables $(\alpha=.69 \mathrm{y} .65$, respectivamente) y el último factor Celos/ Soledad tuvo un valor demasiado bajo $(\alpha=.43)$.

Por otra parte, el cuestionario MESSY en la versión para el profesor tiene un formato muy similar, con algunas variaciones en el número de ítems para las dimensiones antes señaladas y con la diferencia de que la última dimensión está conformada sólo por Asertividad Inapropiada/ Impulsividad, y Soledad/Ansiedad Social.

\section{Procedimiento}

La técnica de intervención implementada fue un taller vivencial; se explicó a los jóvenes el beneficio de participar en él y la libertad de retirarse si en algún momento así lo deseaban. El director del albergue firmó el consentimiento informado. Todos los integrantes del albergue fueron invitados a asistir y la intervención se realizó en un solo grupo. Se aplicó el instrumento de evaluación antes de comenzar la intervención, y al terminar todas las sesiones del taller se llevó a cabo la post-evaluación a siete de los ocho participantes.

El taller estuvo conducido por dos psicólogas. La duración del taller fue de 20 sesiones de una hora y media, con la siguiente estructura: juego de inicio, juego relacionado con el tema, tema, actividad principal, plenaria y convivencia. Las temáticas de las sesiones y sus contenidos fueron las siguientes:

1. Introducción: presentación del taller, actividad para la expresión de expectativas y resolución de dudas que pudieran tener. Establecimiento del reglamento y realización de una manta, con la participación de todos.

2. Conociéndome: tomar conciencia de nuestro cuerpo y de los gustos propios e intereses como parte de nuestra identidad.

3. Yo a través del tiempo: tomar conciencia de los cambios que hemos tenido a lo largo de nuestra vida y favorecer el conocimiento de las propias habilidades, destrezas, virtudes y áreas de oportunidad. 
4. Cómo soy ahora: identificación más profunda de las características personales e integración de la información que hayan ido descubriendo sobre ellos mismos.

5. Conociendo la empatía: presentación de un video con dicha temática e identificación de las acciones empáticas en los personajes de la película.

6. Conociendo a mi amigo: promover y reforzar la confianza grupal mediante el comportamiento de algunos temas.

7. Viendo a través de tus lentes: compartir características personales que tengan en común y poner en práctica la empatía, para fomentar la capacidad de ponerse en el lugar del otro.

8. Aprendiendo sobre la comunicación: importancia de la comunicación y de "comunicarnos bien".

9. Siendo asertivo: aprendiendo a decir "no" a las cosas que no van con nuestros principios o gustos y dar a conocer los derechos asertivos que todas personas tenemos, así como dar ejemplos sobre cómo aplicarlos.

10. ¿Cómo digo lo que quiero decir?: desarrollar la capacidad de reconocer y expresar aspectos positivos a las personas que nos rodean.

11. Recapitulación: recordar lo visto en las sesiones pasadas y conversar sobre su experiencia en las fiestas decembrinas.

12. Yo puedo ser tu amigo, tú puedes ser mi amigo: trabajo en parejas, conocer los valores de la amistad y la capacidad propia para ser un amigo.

13. Aprendiendo a confiar: trabajo en equipo, favorecer el hacer algo "lindo" por otra persona y fomentar la confianza de los integrantes en sus figuras de autoridad y entre ellos como grupo.

14. ¿Qué es una emoción?: aprender sobre las diferentes emociones y su función en nuestra vida diaria.

15. Conociendo mis emociones: aprender a escuchar lo que nuestras emociones nos quieren decir y el impacto que tienen en nuestra vida dependiendo de cómo reaccionemos ante ellas.

16. ¿Qué hago con esto que siento?: aprender a manejar nuestras emociones y saber expresarlas de la mejor manera, además de descubrir la importancia y los beneficios que puede traernos estas habilidades.

17. ¡Estamos en problemas!: conocer qué es un problema y favorecer el ver los problemas como oportunidades de cambio y crecimiento personal y social. Mostrar que en un conflicto, los participantes tienen diferentes visiones.

18. ¿Cómo puedo solucionar un problema?: favorecer la creatividad al momento de resolver un problema y brindarles herramientas útiles al momento de resolver problemas cotidianos.

19. Yo puedo tomar decisiones: favorecer el trabajo en equipo, ofrecer herramientas para evaluar diferentes posibilidades $\mathrm{y}$ 
actuar proactivamente para hacer que las cosas sucedan.

20. Finalización del taller: hacer una recapitulación acerca de lo vivido $\mathrm{y}$ aprendido en el taller, para favorecer el compartir experiencias de lo que más les gustó, lo que no quieren olvidar, etc. Agradecimiento a los participantes de parte de las facilitadoras.

\section{Resultados}

Los resultados que se registraron en el grupo después de la intervención se pueden apreciar a continuación.

En cuanto a los resultados obtenidos de la aplicación de la escala MESSY, se realizaron cuatro comparaciones para una mejor apreciación de los cambios registrados, de acuerdo con las clasificaciones de las escalas.

Como se puede observar en la figura 1 , todos los jóvenes mostraron una puntuación más alta en la dimensión Habilidades Sociales Adecuadas del autoinforme, al terminar el taller. El sujeto siete ya no se encontraba en el albergue cuando se realizó la prueba postest. En cuanto a la segunda dimensión, la figura 2 muestra que no hubo una disminución significativa en general para el grupo, según los autoinformes.

En cuanto a los cuestionarios para el profesor, llenados por el director del albergue, se puede observar, como indica la figura 3 , un aumento para la primera dimensión de la escala, Habilidades Sociales Adecuadas, a excepción de tres jóvenes, los cuales fueron calificados con la misma puntuación al iniciar y al terminar el taller. Para la segunda dimensión observada en la figura 4 (Asertividad Inapropiada/ Impulsividad y Soledad/Ansiedad Social), tampoco se puede observar una disminución significativa en la puntuación.

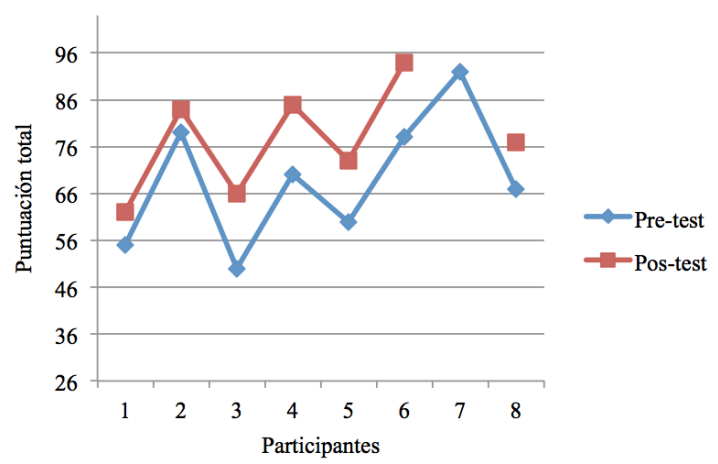

Figura 1. Comparación de la dimensión Habilidades Sociales Apropiadas del Pretest y del Postest en la versión autoinforme.

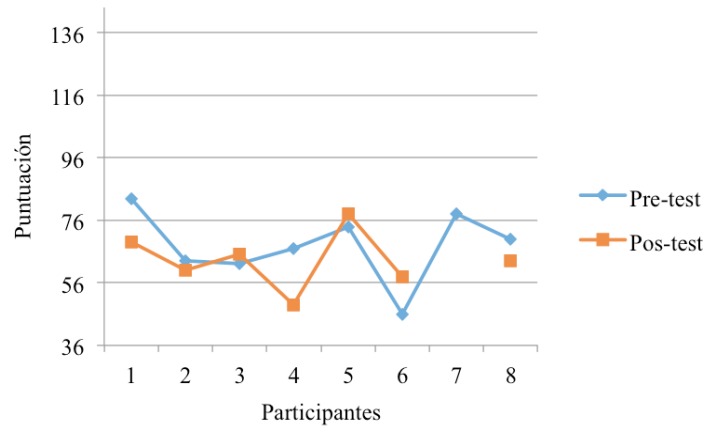

Figura 2. Comparación de la dimensión Asertividad Inapropiada, Impulsividad, Sobreconfianza y Celos/ Soledad del pretest y postest en la versión autoinforme. 


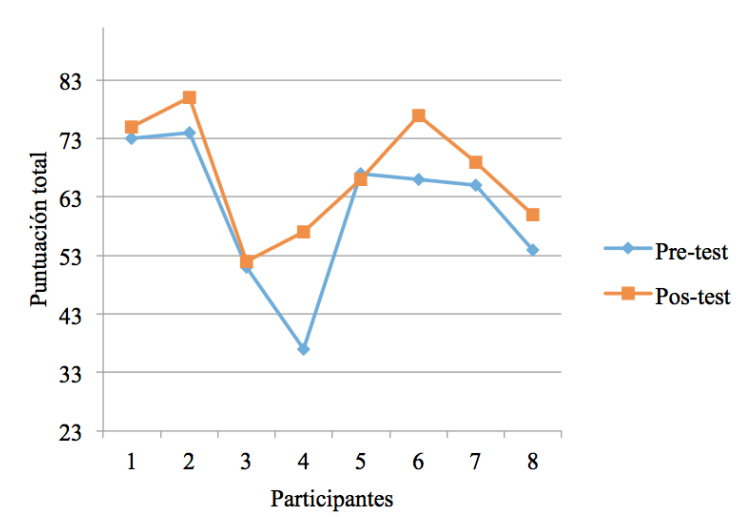

Figura 3. Comparación de la dimensión Habilidades Sociales Apropiadas del pretest y del postest en el cuestionario para el profesor.

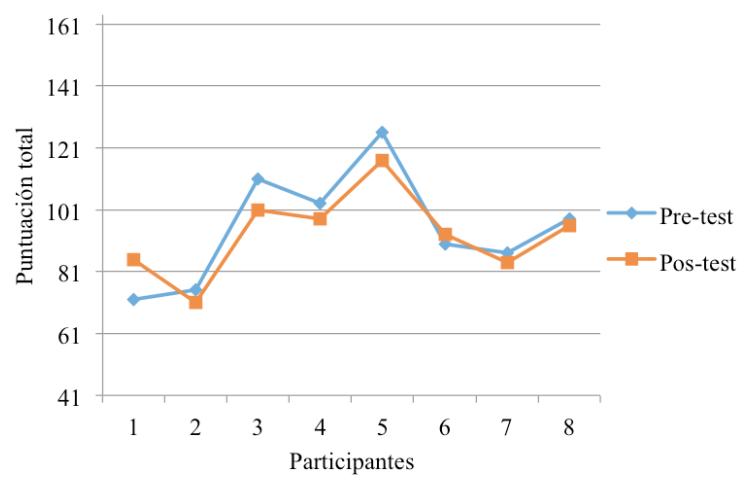

Figura 4. Comparación de la dimensión Asertividad Inapropiada/Impulsividad y Soledad/Ansiedad Social del postest y del pretest en el cuestionario para el profesor.

Los jóvenes mostraron cambios favorables de manera progresiva a lo largo de las sesiones del taller, lo que pudo observarse desde la segunda tercera parte del taller $\mathrm{y}$, más claramente, después de la segunda mitad del taller.
Los adolescentes mejoraron su puntualidad y su constancia para asistir al taller. En las últimas sesiones, todos los jóvenes asistieron al lugar sin la necesidad de ser llamados. Se mostraron participativos en las actividades que se realizaban, daban su opinión y aportaban ideas de manera voluntaria, asimismo, mejoraron significativamente su trabajo, tanto de manera individual como en equipo. Respetaban los turnos, las agresiones entre ellos disminuyeron en demasía y la atención hacia sus compañeros y hacia las facilitadoras aumentó. Inclusive, entre ellos surgió la iniciativa de pedir respeto para la persona que estuviera hablando cuando había quienes se distraían. Su capacidad reflexiva también tuvo un desarrollo favorable, ya que eran capaces de realizar ejercicios de mayor complejidad, de concentrarse en éstos el tiempo necesario y de entregar a las facilitadoras trabajos más ordenados.

Por otra parte, los adultos a cargo de los jóvenes daban buenas referencias de éstos en cuanto a su comportamiento. Las peleas entre compañeros disminuyeron y los conflictos entre los jóvenes y los adultos también fueron de menor frecuencia. La mayoría de los jóvenes realizaba sus labores domésticas sin la necesidad de que se las recordaran muchas veces; sus tareas escolares tenían mejor calidad y su capacidad de concentración en las clases había aumentado. En las ocasiones que hubo eventos en el albergue, los jóvenes se mostraron más participativos e interactuaban mejor con las personas externas al lugar. 


\section{Discusión}

El objetivo de la intervención fue fomentar las HS en los jóvenes de una institución, mediante actividades lúdicas con un enfoque Gestalt. Se cree que el uso del juego fue muy importante en el desarrollo de estas habilidades, ya que el adolescente al realizarla no siente estar obligado a aprender algo, y a la vez está practicando sin darse cuenta estas mismas habilidades, por ejemplo, al respetar turnos en un juego, ponerse de acuerdo en los juegos de equipos, entre otras.

El participar en una intervención grupal con quienes conviven a diario, les permitió mostrarse de una forma más auténtica, de este modo, los cambios que se iban observando se consideraba auténticos también, ya que la posibilidad de mostrarse inhibidos ante el grupo o dirigir su comportamiento para agradar a los demás, eran menores. De igual forma, se mostraron más cómodos al realizar las actividades en su grupo y esto les brindó mayor oportunidad de aprender de las interacciones sociales entre ellos mismos.

El trabajoconadolescentes institucionalizados es un campo que los profesionales de la salud mental no debemos descuidar. Existen muchas áreas que se pueden y se deben trabajar con esta población, además de la socialización. Por lo que respecta a este trabajo, se pudo observar los beneficios obtenidos por los jóvenes al trabajar en grupo para el fomento de las HS. Por otra parte, es importante también buscar disminuir las conductas de riesgo o conductas que no favorezcan la socialización de los jóvenes, ya que sólo fomentar las HS no disminuye significativamente otro tipo de conductas, motivo por el cual es decisivo prestar atención a esta área.

Se cree que la constancia de las facilitadoras y la duración del taller, 20 sesiones, tuvieron una repercusión positiva en los jóvenes, dado que el esquema de abandono presente en adolescentes institucionalizados puede dificultar la relación con las figuras de autoridad en el contexto donde se desenvuelven. Por esto, al inicio eran más notorias las conductas de prueba del vínculo de los jóvenes hacia las facilitadoras, pero durante el progreso de las sesiones fueron disminuyendo; la actitud mostraba se relaciona con los cambios positivos que presentaron los adolescentes para la finalización de la intervención.

Las sesiones con juego son comúnmente utilizadas para trabajar con niños pequeños $y$ pre-púberes, en los talleres psicoeducativos se han visto más en el trabajo con adolescentes. Sin embargo, las actividades lúdicas demostraron ser bien recibidas por los adolescentes y fueron clave para la asistencia de los jóvenes al taller. Los jóvenes mostraron un interés considerable en participar en los juegos más activos $\mathrm{y}$ dinámicos, y fue mediante el juego que se empezaron a ser más evidentes los cambios.

La investigación presentó, empero, algunas limitantes importantes, como el hecho de carecer de mayor información sobre los jóvenes para poder relacionar los resultados con otros factores y no sólo con la institucionalización. Incluso, la misma institucionalización fue una limitante para el trabajo con los jóvenes, ya que 
no se pudo disponer de otros ambientes para las sesiones y práctica de las HS. Tampoco se creó un espacio para trabajar con los cuidadores de los adolescentes, por diversas razones, a pesar de lo importante que resulta el trabajo paralelo del menor y del adulto para generar cambios más congruentes y duraderos.

Si bien las características de la investigación y de la población elegida, evitan generalizar los resultados, se pueden hacer futuras investigaciones que tomen en cuenta las limitaciones de las pasadas. Así, se pueden realizar estudios longitudinales para observar la trayectoria de más niños y adolescentes institucionalizados, además de realizar grupos de control que generen un punto de comparación y de este modo enriquecer la investigación.

\section{Referencias}

Burga, R. (1981). Terapia gestáltica. Revista Latinoamericana de Psicología, 13(1), 85-96. Caballo, V. (2010). Manual de evaluación y entrenamiento de las habilidades sociales $\left(6^{\mathrm{a}}\right.$ ed.). Madrid: Siglo XXI.

Carcelén, M., \& Martínez, P. (2008). Perspectiva temporal futura en adolescentes institucionalizados. Revista de Psicología, 16(2), 255-276.

Fernández, M., \& Fernández, A. (2012).

Problemas de comportamiento y competencias psicosociales en niños $\mathrm{y}$ adolescentes institucionalizados. Universitas Psychologica, 12(3), 797-810.
Ipiña, M., Molina, L., \& Reyna, C. (2011). Propiedades psicométricas de la Escala MESSY (versión autoinforme) en niños argentinos. Revista de Psicología, 29(2), 245-264.

Ison, M., \& Morelato, G. (2007). Habilidades socio-cognitivas en niños con conductas disruptivas y víctimas de maltrato. Universitas Psychologica, 7(2), 357-367.

Kelly, J. (2002). Entrenamiento de las habilidades sociales. Bilbao: D. D. B.

Lacunza, A. (2009). Las habilidades sociales como recursos para el desarrollo de fortalezas en la infancia. Psicodebate: Psicología, Cultura y Sociedad, 10(1), 231-248.

Lacunza, A., \& Cortini, N. (2011). Las habilidades sociales en niños y adolescentes. $\mathrm{Su}$ importancia en la prevención de trastornos psicopatológicos. Fundamentos en Humanidades, 3(23), 159-182.

Matson, J. L., Rotatori, A. F., \& Helsel, W. J. (1983). Development of a rating scale to measure social skills in children: The Matson Evaluation of Social Skills with Youngsters (MESSY). Behavior Research Therapy, 21(49), 335-340.

Oaklander, V. (2001). Ventanas a nuestros niños (6 $6^{\mathrm{a}}$ ed.). Santiago de Chile: Editorial Cuatro Vientos.

Oaklander, V. (2008). El tesoro escondido. La vida interior de niños y adolescentes. Santiago de Chile: Editorial Cuatro Vientos. 
Ostrovsky, G. (2007). Cómo construir competencias en los niños y desarrollar su talento 2. Bogotá: D’Vinni S. A.

Romera, E., Ortega, R., \& Monks, C. (2008). Impacto de la actividad lúdica en el desarrollo de la competencia social. International Journal of Psychology and Psychological Therapy, 8(2), 193-202.

Schaefer, C. (2012). Fundamentos de terapia de juego ( $2^{\mathrm{a}}$ ed.). México: Manual Moderno.

Schaffer, R. (2000). Desarrollo social. México: Siglo Veintiuno.

Trianes, M. V., Blanca, M. J., Muñoz, A., García, B., Cardelle-Elawar, M., \& Infante, L. (2002). Relaciones entre evaluadores de la competencia social en preadolescentes: Profesores, iguales y autoinformes. Anales de Psicología, 18(2), 197-214.

Trianes, M., Muñoz, A., \& Jiménez, M. (1997). Competencia social: su educación y tratamiento. Madrid: Pirámide.

Recibido el 5 de abril de 2016

Revisado el 12 de mayo de 2016

Aceptado el 28 de julio de 2016 